\section{Magneto-optical polymer}

Opt. Lett. 43, 4615-4618 (2018)

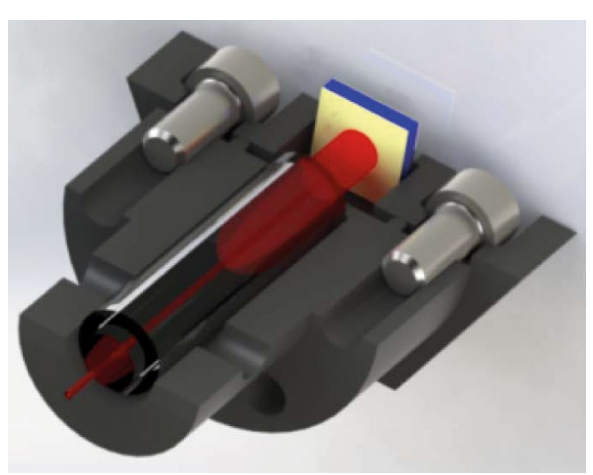

Credit: OSA

A compact, highly sensitive all-optical magnetometer has been demonstrated by a United States-Belgium collaboration. The device is based on a magneto-optical material with a large Verdet constant $\left(10^{6}\right)$ - a polymer film containing dysprosium $\left(D y^{3+}\right)$-doped magnetite and cobalt ferrite nanoparticles - that is placed in a fibreoptic Sagnac interferometer. The presence of a magnetic field causes a Faraday rotation of the polarization of the 1,310-nm laser beam as it passes through the polymer film and the resulting interference signal is measured by a balanced detector. The team says that the device has a sensitivity of $20 \mathrm{fT} \mathrm{Hz}^{-1 / 2}$ and is potentially well suited to biomedical applications as it is not only small (around an inch long) and sensitive, but also operates at room temperature, does not require shielding and is highly robust against noise. As a demonstration of its applicability, the team showed that the sensor can detect the magnetic field associated with the beat of the human heart. In principle, it should be able to detect the tiny (50-500 fT) magnetic fields associated with neural activity in the brain. $\quad O G$

https://doi.org/10.1038/s41566-018-0295-0

\section{OPTOMECHANICS}

\section{Anti-damped nanobeam \\ ACS Photon. https://doi.org/cvsj (2018)}

On-chip optical sensors based on optomechanical resonances are potentially attractive for refractive index sensing for chemical, biological, medical and even environmental analysis, yet greater precision is still desired. Fei Pan and colleagues at Tsinghua University, Beijing, have now demonstrated an integrated optomechanical cavity based on a silicon nanobeam structure that they say exhibits a record (in an ambient environment) mechanical quality factor of 18,300 . This mechanical quality factor is predicted to allow sensing with a sensitivity on the order of $10^{-7}$, which the authors say is an order of magnitude higher than that of conventional silicon-based approaches. The improvement is due to radiation-pressure antidamping; in particular, the linewidth of the mechanical resonance is effectively reduced by optical forces. The structure is a 556-nm-wide and 220-nm-tall Si beam with periodic hole-array mirrors containing a central defect. It should be noted that the authors have not yet conducted a sensing experiment and that the sensitivity was inferred.

DFPP

https://doi.org/10.1038/s41566-018-0292-3

\section{RANDOM LASERS}

\section{Soliton assistance \\ Nat. Commun. 9, 3863 (2018)}

Although random lasers are attractive due to their design flexibility, the lack of a well-defined, directional output beam hinders their use in some applications.

\title{
NONLINEAR OPTICS
}

\section{Atomically thin materials}

Placing a 2D material in front of a partially reflecting broadband mirror can dramatically enhance the nonlinear interaction between individual photons, according to a US team of researchers. In a theoretical study, Dominik Wild and co-workers, from Harvard University and the University of Connecticut, discovered that a transition metal dichalcogenide (TMD) monolayer acts as a perfect reflector even though it is thinner than a wavelength. However, when the distance between the TMD monolayer and the mirror is close to half-integer multiples of the exciton resonance wavelength, perfect transmission is achieved. The phenomenon is similar to blockade-type effects that occur in quantum-well cavities, where the presence of a single polariton blocks subsequent photons from entering the cavity.

https://doi.org/10.1038/s41566-018-0294-1

Now, Sreekanth Perumbilavil and colleagues from Finland, Italy, the UK and the US have demonstrated a soliton-enhanced random laser that combines highly efficient operation $(9.62 \%)$ with a smooth spatial emission profile and high directionality. The team exploit weakly scattering dye-doped nematic liquid crystals (NLCs) that exhibit random lasing but also support propagation of self-guided optical spatial solitons. Specifically, they used an NLC called E7 doped with $0.3 \mathrm{wt} \%$ of the dye pyrromethene 597 in planar cells. To generate a spatial soliton, a continuous-wave laser beam at $1,064 \mathrm{~nm}$ was launched in a 100 - $\mu \mathrm{m}$-thick, 2-mm-long planar cell containing the dye-doped NLC. The team showed that the direction of emission can be controlled by voltage or other external stimuli, and that the laser can be switched on and off by all-optical means.

https://doi.org/10.1038/s41566-018-0296-Z

\section{ELECTRO-OPTICS}

\section{Subcycle control}

Science 361, 1358-1363 (2018)

Mode-locked cavities have long been exploited where laser pulses with high stability or precision are required, such as in optical atomic clocks. However, electrooptic modulation arguably offers higher repetition rates than mode-locking and could thus lead to increased precision. Now, David Carlson and co-workers at the National Institute of Standards and Technology and the University of Colorado in Boulder, Colorado, report electro-optic generation of $100-p J$ pulses at rate of 30 $\mathrm{GHz}$ and also show sub-optical-cycle timing precision. The total output power is about $1.1 \mathrm{~W}$ contained in spectra that span from $750 \mathrm{~nm}$ to about $2,700 \mathrm{~nm}$ in wavelength. A 1,550 -nm continuous-wave laser was phaseand intensity-modulated by a commercial 10-GHz dielectric resonant oscillator locked to a microwave cavity. The set-up involves an intensity modulator in series with multiple phase modulators in order to create a deterministic cascade of sidebands (comb lines). Broadband thermal noise, a characteristic of electro-optic modulation, is optically filtered by a Fabry-Pérot cavity, and spectral broadening then takes place in a nonlinear fibre. Analysis confirms that the pulses are up to $15 \mathrm{ps}$ in duration (2.8 optical cycles).

https://doi.org/10.1038/s41566-018-0293-2

Oliver Graydon, Noriaki Horiuchi, David F. P. Pile and Rachel Won 